\title{
LA PROVA STATISTICA NEL PROCESSO PENALE
}

\author{
Nota del m.e. VITTORIO B. FROSINI (*)
}

(Adunanza del 24 ottobre 2013)

SuNTO. - I primi tre paragrafi sono dedicati all'esposizione di temi generali, e cioè il ruolo delle probabilità oggettive e delle probabilità soggettive nei processi, la procedura per la determinazione di probabilità condizionali, e la formula (o teorema) di Bayes; quest'utimo argomento concerne lo strumento fondamentale che permette il calcolo delle probabilità delle possibili cause (che possono avere agito), data l'informazione posseduta su dati effetti riscontrati. Strettamente collegata con l'applicazione del teorema di Bayes è la c.d. «fallacia del condizionale trasposto», la cui applicazione porta usualmente a una errata attribuzione di colpevolezza (per un dato reato). Il quinto paragrafo è dedicato a una discussione di alcune principali caratteristiche di due famosi processi, quelli di Alfred Dreyfus in Francia e di Sally Clark nel Regno Unito, nei quali la predetta fallacia può essere riconoscibile. Il paragrafo conclusivo presenta tipi di processi in cui in cui è richiesto un giudizio relativamente a gruppi di persone; i metodi statistici usualmente applicati in questi casi discendono dai c.d. test di significatività.

$* * *$

ABSTRACT. - A general topic is faced in the first three sections, i.e. the role of objective and subjective probabilities in trials, the determination of conditional probabilities, and Bayes' formula (or theorem); the last topic is a fundamental device which allows the computation of probabilities of possible causes, given related information about certain effects. Strictly connected to this argument is the «fallacy of the transposed conditional», which usually implies an unwarranted assumption of guilt. The fifth section is devoted to a discussion of some main features of two famous trials, those of Alfred Dreyfus in France and of Sally Clark in U.K., in which the above fallacy is clearly recognized. The final section presents kinds of trials which require a judgment about groups of people; the statistical methods applied in these cases pertain to the so-called tests of significance.

(") Dipartimento di Scienze Statistiche dell'Università Cattolica del Sacro Cuore, Milano, Italy. E-mail: benito.frosini@unicatt.it 


\section{Probabilità oggettiva e Probabilità SOgGettiva}

La probabilità di un evento casuale è oggettiva quando trova una generale adesione, e quindi è indipendente dal soggetto valutatore. Casi tipici sono dati dalle probabilità associate agli esiti di giochi di sorte, dove gli eventi elementari (cioè i singoli eventi, non ulteriormente scomponibili, dello stesso gioco o esperimento) sono equiprobabili (ad es. usuali giochi di carte, lanci di dadi e monete, gioco della roulette); dall'esame di questi giochi di sorte è nato - intorno alla metà del secolo XVII - il moderno Calcolo delle probabilità. Per questi giochi, e per loro generalizzazioni (anche per eventi elementari non equiprobabili), si parla in genere di esperimenti casuali (cfr. Frosini, 2009, Cap. 7)

La probabilità di un evento casuale è invece soggettiva quando la sua valutazione può variare da individuo a individuo: l'evento non dipende dalla realizzazione di un esperimento casuale, oppure l'esperimento casuale non è completamente noto. Un caso particolare, notevole per i processi civili e penali, è dato dai c.d. eventi unici: ad essi può essere associata soltanto una probabilità soggettiva. Naturalmente tale probabilità deve risultare dalla sintesi di tutte le informazioni possedute dal soggetto valutatore intorno alla possibilità di accadimento del dato evento. Nella letteratura giuridica, invece che di probabilità soggettiva, si parla solitamente di probabilità logica. Come si è già detto più sopra, il ricorso alla probabilità soggettiva è inevitabile nel caso di eventi unici, che non sono prodotti da un esperimento casuale, o per i quali l'eventuale esperimento casuale generatore è ignoto.

Per quanto le probabilità utilizzate in un giudizio civile o penale debbano essere - come è ovvio - possibilmente oggettive, e quindi condivise da tutte le parti in causa, conviene avvertire fin da ora che la probabilità finale del giudice, in un giudizio civile o penale, è inevitabilmente una probabilità soggettiva (ovvero logica); essa deve però essere sostenuta - per essere convincente - da un ragionamento il più possibile oggettivo.

\section{Probabilità CONDIZIONALE E CAUSA PROBABILISTICA}

La grande maggioranza delle probabilità rilevanti in un processo sono probabilità condizionali: si tratta di probabilità relative a eventi condizionati. Ad es., con riferimento alla estrazione di una carta da un 
mazzo di 40 carte, la probabilità dell'evento $A=$ "estrazione di un Re" è data dal rapporto $4 / 40=1 / 10$ (applicando la regola "casi favorevoli diviso casi possibili") per l'esperimento casuale ipotizzato; se invece si possiede l'ulteriore informazione che la carta estratta è una figura, il c.d. spazio campionario (cioè l'insieme degli eventi elementari che possono accadere) viene ristretto alle 12 figure presenti nel mazzo di carte: in tal caso la probabilità di avere estratto un Re - sempre applicando il criterio "casi favorevoli diviso casi possibili" - è 4/12=1/3 (cfr. Frosini, 2009, Cap. 7).

Per l'applicazione di una formula generale, che sarà indicata nel seguito, dobbiamo indicare anche un simbolo per l'evento condizionante; nel caso dell'esempio l'evento condizionante è $B=$ "estrazione di una figura". Con il simbolo $A \mid B$ (che si legge " $A$ dato $B$ ) si intende l'evento $A$ condizionato dall'evento $B$ (ovvero, tutti gli eventi elementari che si possono concretamente realizzare sono soltanto quelli compresi in $B$ ). Nel caso dell'esempio $A \mid B$ ha il significato già associato, e cioè "estrazione di un Re da carte che sono figure".

Formalmente, se indichiamo in generale con $P(A)$ e $P(B)$ le probabilità dei due eventi (riferite all'esperimento casuale originario), entrambe $>0$, e con $P(A \& B)$ la probabilità che i due eventi $A$ e $B$ accadano entrambi, la probabilità che l'evento $A$ accada sotto la validità della condizione $B$ si scrive (e si legge "probabilità di $A$ dato $B$ ")

$$
P(A \mid B)=\frac{P(A \& B)}{P(B)}
$$

N.B. L'evento $A \& B$ può essere impossibile, e in tal caso è correttamente $P(A \mid B)=0$.

Nei processi, e anche in molte inferenze della vita reale, si fa solitamente riferimento a frasi, o proposizioni, e a relazioni di implicazione fra dette proposizioni (del tipo usuale "se $a$ allora $b$ ", dove $a$ e $b$ sono proposizioni). L'implicazione logica aristotelica, solitamente indicata con $a \Rightarrow b$, è di tipo deterministico, e significa che $a$ è condizione sufficiente per $b$, mentre $b$ è condizione necessaria per $a$. Può però accadere, in moltissimi casi, che "dato $a$ " la condizione $b$ non si realizzi sempre, ma solo in una data frazione dei casi; si può pertanto associare a tale implicazione una data probabilità. Se con $A$ e $B$ indichiamo le frazioni di individui di una data popolazione che soddisfano - rispettivamente - le condizioni $a$ e $b$, la probabilità dell'implicazione $a \Rightarrow b$ è definita come segue: 
$P(a \Rightarrow b)=P(B \mid A)=\frac{P(A \& B)}{P(A)}$.

Ad es. possiamo essere interessati a una implicazione relativa a una popolazione di individui che lavorano in un dato ambiente, dove $a=$ "lavora nel dato ambiente", e $b=$ "sviluppa un tumore polmonare".

E' importante osservare che anche in presenza di causa probabilistica è possibile che l'inferenza sia certa, nel senso che la probabilità della condizione contingentemente necessaria può essere uguale a uno.

Esempio:

$P(a \Rightarrow b)=P(B \mid A)=0,01$

$\mathrm{ma}$

$P(b \Rightarrow a)=P(A \mid B)=1$

in tal caso, dato l'evento $A$, l'evento $B$ accade raramente (una volta su cento, in media), ma accade solo in presenza di $A$. Naturalmente, per potere applicare questo tipo di inferenza (c.d. legge universale) dobbiamo essere certi della validità di quest'ultima condizione; se riteniamo possibile che l'evento $B$ possa essere provocato da una causa diversa da $A$, anche se non completamente identificata, l'inferenza certa non è più proponibile (Frosini, 2002, pp. 45-46).

\section{Probabilità delle Cause, DATI GLi eFFetTi. FORMULA DI BAYES}

In molti processi vi è l'interesse a risalire, da un fatto noto $B$ (effetto) alla causa che lo ha generato. Si ammetta, per fissare le idee, che le possibili cause - oggetto di indagine - siano $k$, indicate per comodità con $A_{1}, A_{2}, \ldots, A_{k}$ (il problema generale della pluralità delle cause è trattato da Stella, 2001). Che un certo effetto possa essere provocato da cause differenti è di solito pacificamente ammesso; si osservi però che l'asserita pluralità delle cause è incompatibile con l'inferenza deduttiva, come viene tradizionalmente configurata, in cui la causa è intesa come condizione necessaria e sufficiente per l'effetto (cfr. Copi, 1964, p. 407). 
Se ci può essere pluralità di cause, allora non sono possibili inferenze (deduttive) dagli effetti alle loro cause. Quanto alla apparente pluralità delle cause, vale che, se un effetto viene specificato con molta precisione, l'apparente pluralità delle cause solitamente scompare. Vale comunque, deduttivamente, che se si possiedono sufficienti informazioni per poter escludere tutte le possibili cause meno una, quella rimasta è sicuramente la causa cercata.

Nel seguito si ammetterà di non possedere informazioni tali da portare alla individuazione di una specifica causa; per l'effetto $B$ restano quindi possibili le cause $A_{1}, A_{2}, \ldots, A_{k}$. Allo scopo di eseguire una inferenza induttiva dall'effetto $B$ a ciascuna delle sue possibili cause, è però necessario possedere - e utilizzare - un altro tipo di informazione, e cioè un'informazione di tipo probabilistico. Conviene osservare innanzitutto che le diverse cause hanno in genere una capacità piuttosto differenziata di produrre l'effetto considerato; ad esempio la causa $A_{1}$ è molto efficiente per produrre l'effetto $B$, mentre la presenza di $A_{2}$ è in grado di produrre $B$ solo in una frazione limitata di casi. E' quindi essenziale associare a ciascuna causa un indice sintetico che informi sulla efficienza della stessa causa nella produzione dell'effetto $B$; tale indice sintetico è la probabilità di $B$ dato $A_{r}($ per $r=1,2, \ldots, k)$ :

$P\left(B \mid A_{r}\right)=L\left(A_{r}\right) \quad r=1,2, \ldots, k$

anche chiamata verosimiglianza (likelihood) di $A_{r}$ per $B$.

$\mathrm{Si}$ intuisce come le verosimiglianze abbiano un ruolo importantissimo nella c.d. inferenza statistica. Se ad esempio le possibili cause sono soltanto due $\left(A_{1}\right.$ e $\left.A_{2}\right)$, e le verosimiglianze stanno nel rapporto di uno a mille $\left(L\left(A_{2}\right) / L\left(A_{1}\right)=1000\right)$, sembra ragionevole indurre che abbia agito la causa $A_{2}$. Ma si intuisce anche che il ragionamento induttivo non è completo: infatti può essere che la realizzazione della causa $A_{2}$ sia molto rara, mentre al contrario sia piuttosto comune la realizzazione della causa $A_{1}$. Si pensi ad esempio a due malattie di gravità molto differenziata, che possono dare luogo allo stesso sintomo $B$, presenti in frazioni molto diverse della popolazione. E' quindi importante tener conto, nel ragionamento induttivo, anche della probabilità delle cause, dette anche probabilità a priori

$P\left(A_{r}\right) \quad r=1,2, \ldots, k$;

più una causa è probabile, più deve essere elevata (a parità di altre con- 
dizioni) la probabilità che l'effetto sia dovuto alla data causa. La stessa intuizione porta quindi a definire $P\left(A_{r} \mid B\right)$ (probabilità della causa $A_{r}$ dato l'effetto $B$ ) come proporzionale al prodotto della probabilità a priori di $A_{r}$ per la verosimiglianza di $A_{r}$ :

$P\left(A_{r} \mid B\right) \propto P\left(A_{r}\right) \times P\left(B \mid A_{r}\right) \quad r=1,2, \ldots, k$.

La formula completa, comprendente il coefficiente di proporzionalità, chiamata "formula di Bayes" (da Thomas Bayes, 1702-1761) è la seguente (Frosini, 2009, p. 205):

$$
\begin{aligned}
& P\left(A_{r} \mid B\right)=\frac{P\left(A_{r}\right) \times P\left(B \mid A_{r}\right)}{P(B)} \quad r=1,2, \ldots, k \\
& P(B)=\sum_{r=1}^{k} P\left(A_{r}\right) \times P\left(B \mid A_{r}\right) .
\end{aligned}
$$

Questa formula deriva molto semplicemente dalla formula generale della probabilità condizionale

$$
P\left(A_{r} \mid B\right)=\frac{P\left(A_{r} \& B\right)}{P(B)} \text {; }
$$

formalmente, questa formula permette di scambiare i due eventi $A_{r}$ e $B$ rispetto alla formula della verosimiglianza $P\left(B \mid A_{r}\right)$ : dalla probabilità dell'effetto $B$ data la causa $A_{r}$ si passa alla probabilità della causa $A_{r}$ dato l'effetto $B$.

I confronti fra diverse probabilità del tipo $P\left(A_{r} \mid B\right)$ sono facilitati quando essi vengano effettuati tramite rapporti del tipo (dove si considerano, a titolo esemplificativo, le cause $A_{1}$ e $A_{2}$ ):

$$
\frac{P\left(A_{1} \mid B\right)}{P\left(A_{2} \mid B\right)}=\frac{P\left(A_{1}\right)}{P\left(A_{2}\right)} \times \frac{P\left(B \mid A_{1}\right)}{P\left(B \mid A_{2}\right)} ;
$$

nel rapporto il denominatore comune $P(B)$ viene eliminato, e lo stesso rapporto si presenta come il prodotto di due rapporti, fra le probabilità a priori e fra le verosimiglianze.

Una particolare applicazione del suddetto rapporto riguarda i due eventi contrari rilevanti in molti processi, in cui $A_{1}=\bar{G}$ (guilty, cioè un dato individuo è colpevole) e $A_{2}=\bar{G}$ (not guilty, cioè non colpevole); in questo tipo di applicazione l'effetto $B$ è solitamente indicato con $E$, intendendo che l'osservazione $E$ consiste nell'evidenza fattuale presen- 
tata nel corso del processo. In tal caso il suddetto rapporto si presenta nella forma c.d. odds ratio:

$$
\frac{P(G \mid E)}{P(\bar{G} \mid E)}=\frac{P(G)}{P(\bar{G})} \times \frac{P(E \mid G)}{P(E \mid \bar{G})}
$$

che eguaglia il prodotto odds ratio fra le probabilità a priori (o probabilità iniziali) e il rapporto di verosimiglianza. In sostanza, il rapporto di verosimiglianza trasforma il precedente rapporto tra le probabilità iniziali nel rapporto tra le probabilità finali (cioè tenuto conto della informazione E). A seguito della moltiplicazione per il rapporto di verosimiglianza, che può essere $>=<1$, il rapporto fra le probabilità iniziali può aumentare (a favore della colpevolezza), oppure restare costante, oppure diminuire.

Le probabilità soprascritte, cioè le probabilità iniziali $P\left(A_{r}\right)$ e le verosimiglianze $P\left(E \mid A_{r}\right)$ possono essere - nelle particolari applicazioni probabilità oggettive o soggettive (o anche essere graduate, fra oggettive pure e soggettive pure, fra i due limiti concettuali). In molte applicazioni le verosimiglianze hanno natura oggettiva o quasi oggettiva; al contrario, le probabilità iniziali $P\left(A_{r}\right)$ hanno quasi sempre natura eminentemente soggettiva (anche se possibilmente basate su informazioni attendibili riguardo all'evidenza portata nel processo). Questo accade per il fatto che nelle più usuali applicazioni tali probabilità si riferiscono a eventi unici; come già accennato in precedenza, gli eventi cui si riferiscono tali probabilità non possiedono la proprietà di essere ricompresi - come eventi casuali - in uno spazio campionario formalmente definito (Frosini, 2009, p. 190). In questi casi, piuttosto comuni, tali probabilità riflettono essenzialmente il grado di fiducia (fra 0 e 1 ) che un dato individuo associa all'accadimento di un certo evento ( $\mathrm{ad}$ es. $A_{r}$ o anche $E \mid A_{r}$ ).

Come conclusione (provvisoria) riguardo alla applicazione dell'approccio bayesiano a una inferenza induttiva, possiamo elencare quali assunzioni e quali informazioni sono necessarie per l'applicazione formale della formula di Bayes:

(1) dobbiamo essere sicuri che le possibili cause $A_{1}, A_{2}, \ldots, A_{k}$ dell'evidenza $E$ esauriscono l'insieme delle possibili cause; nelle applicazioni ai processi penali ciò è formalmente assicurato quando si passi al riferimento a due sole cause, cioè $G$ (colpevole) e $\bar{G}$ (innocente);

(2) devono essere note tutte le probabilità a priori - o probabilità iniziali $-P\left(A_{r}\right), r=1,2, \ldots, k$, che usualmente sono probabilità soggettive, quindi possono variare da individuo a individuo; 
(3) dobbiamo conoscere tutte le verosimiglianze $P\left(E \mid A_{r}\right), r=1,2, \ldots, k$, che possono essere probabilità oggettive o soggettive.

Con queste informazioni è possibile calcolare le probabilità finali (o probabilità a posteriori) $P\left(A_{r} \mid E\right)$, in applicazione della formula di Bayes. Queste probabilità sono in genere di natura soggettiva, ma possono essere valutate come sostanzialmente oggettive se molte probabilità a priori e molte verosimiglianze, che entrano nel calcolo, hanno natura prevalentemente oggettiva.

\section{LA FALLACIA DEL CONDIZIONALE TRASPOSTO}

In letteratura si usa indicare come "fallacia del condizionale trasposto" una vera e propria fallacia logica, meno nota delle fallacie ricomprese nella logica tradizionale risalente ad Aristotele, in quanto richiede gli strumenti (anche se si tratta degli strumenti più elementari) del Calcolo delle probabilità, che si è sviluppato a partire dalla metà del XVII secolo. Gli effetti di tale fallacia, purtroppo riconoscibile anche in alcuni processi effettivamente realizzati, sono tuttavia devastanti, per cui tale fallacia deve essere riconosciuta e temuta (Aitken \& Taroni, 2004, p. 79).

Con riferimento alla simbologia sopra introdotta, sia $E$ l'evidenza (effetto) disponibile negli atti processuali, $G$ l'evento che un dato individuo sia colpevole (guilty) e $\bar{G}$ l'evento che lo stesso individuo sia innocente (not guilty). Le due probabilità $P(\bar{G} \mid E)$ e $P(G \mid E)$ sommano a uno, in quanto si riferiscono a eventi complementari. Fissiamo l'attenzione sulla probabilità $P(\bar{G} \mid E)=$ probabilità di innocenza data l'eviden$\mathrm{za}, \mathrm{da}$ cui eventualmente ricavare la probabilità di colpevolezza data l'evidenza $P(G \mid E)=1-P(\bar{G} \mid E)$. E' essenziale che tale probabilità $P(\bar{G} \mid E)$ non debba essere confusa, ovvero sostituita, con la probabilità $P(E \mid \bar{G})$, che è la probabilità che l'evidenza $E$ sia stata prodotta da un individuo innocente. Nel caso che $E$ e $\bar{G}$ vengano scambiati ne risulta la fallacia del condizionale trasposto: ad esempio, il fatto che raramente l'evidenza $E$ sia prodotta da un individuo innocente, viene interpretato come probabile indicazione di colpevolezza.

Ad esempio, si ammetta di conoscere il gruppo sanguigno di un individuo XY che è stato visto con la vittima alcune ore prima del delitto, e di sapere che è di tipo $E$ (per ipotesi, piuttosto raro). Ciò può cambiare la posizione di XY da vagamente sospettato a probabilmente col- 
pevole se si applica il seguente (pseudo) ragionamento: è piuttosto raro che l'evidenza $E$ venga osservata su un individuo innocente; è quindi improbabile che XY sia innocente. Formalmente, da $P(E \mid \bar{G})=0,01$ si ricava $P(\bar{G} \mid E)=0,01$, e quindi, per l'evento complementare $G, P(G \mid$ $E)=0,99$.

Continuando l'esempio, che sia «piuttosto raro che l'evidenza $E$ venga osservata su un individuo innocente» è un fatto, essendo tale probabilità dell'uno per cento, ma da ciò non deriva minimamente la conclusione dello pseudo-ragionamento. In realtà, dalla sola conoscenza della probabilità $P(E \mid \bar{G})$ non si può ricavare alcuna informazione sulla probabilità $P(\bar{G} \mid E)$; ad esempio, una delle due probabilità può essere prossima a 1 , e l'altra prossima a 0 . Tenuto conto della formula della probabilità condizionale, possiamo scrivere

$P(\bar{G} \& E)=P(\bar{G}) \times P(E \mid \bar{G})$

e quindi

$P(\bar{G} \mid E)=\frac{P(\bar{G} \& E)}{P(E)}=P(E \mid \bar{G}) \times \frac{P(\bar{G})}{P(E)} ;$

$P(\bar{G} \mid E)$ si può quindi ottenere da $P(E \mid \bar{G})$ dopo moltiplicazione per il rapporto $P(\bar{G}) / P(E)$. Proseguendo nella discussione dell'esempio, se la popolazione di riferimento (composta da coloro che almeno teoricamente possono essere colpevoli del delitto) è piuttosto grande, le probabilità di osservare l'evidenza $E$ su un individuo estratto dall'intera popolazione, o dall'intera popolazione dopo avere escluso il colpevole, sono praticamente coincidenti: $P(E) \approx P(E \mid \bar{G})$; con buona approssimazione si ha quindi $P(\bar{G} \mid E) \approx P(\bar{G})$, cosa che ci permette di affermare che gli eventi $\bar{G}$ ed $E$ sono praticamente indipendenti, ovvero che la conoscenza dell'evidenza $E$ non fornisce praticamente alcuna informazione (da sola e nella situazione ipotizzata) nella ricerca del colpevole.

\section{IL PROBLEMA DELLA FALLACIA NEL CASO DI PROBABILITÀ MOLTO PICCOLE}

Come probabilmente il lettore ha intuito dalla presentazione della fallacia del condizionale trasposto al precedente paragrafo, il rischio di 
commettere tale fallacia è maggiore quando le probabilità in discorso sono molto piccole ("è piccola la probabilità che il dato evento sia stato provocato da un individuo innocente, quindi ..."). Rafforzeremo tale concetto con riferimento a due celebri processi.

Il caso Alfred Dreyfus - Una delle "prove" portate contro Alfred Dreyfus nel primo processo (1894) fu quella che valutava come molto piccola la probabilità di composizione delle lettere dell'alfabeto nel c.d. bordereau imputato a Dreyfus (che sarebbe stato da lui inviato all'ambasciata tedesca). Era vero. Ma l'esperto del tribunale, Sig. Bertillon, commise due errori. Primo errore: Non veniva rilevato il fatto che tutte le composizioni di lettere, in una pagina di prosa francese, ha una probabilità molto piccola (dato che i possibili eventi sono in numero grandissimo). Osservazione generale: Se un evento viene caratterizzato da numerose coordinate temporali e spaziali, la sua probabilità è generalmente microscopica $\left(<10^{-6}\right)$. Tutto quello che accade, se caratterizzato con grande precisione, è praticamente impossibile! (c.d. principio della irripetibilità degli esperimenti). Secondo errore: Si tratta di un lampante esempio della fallacia del condizionale trasposto: avendo osservato che $P(E \mid \bar{G})=$ probabilità di osservare l'evidenza $E$ sotto l'ipotesi che il documento non sia stato contraffatto, è molto piccola, si è dedotto erroneamente che è molto piccola la probabilità $P(\bar{G} \mid E)$, cioè che Dreyfus è innocente data l'evidenza, e quindi che è grande la probabilità di colpevolezza.

Il caso Sally Clark - Il primo figlio di Sally Clark morì improvvisamente all'età di tre mesi, mentre la madre era l'unica altra persona presente in casa (anno 2001). Il caso fu archiviato come SIDS (Sudden Infant Death Syndrome). Il secondo figlio di Sally Clark morì in circostanze strettamente analoghe. Essa fu arrestata, e incolpata dell'omicidio di entrambi i figli; nel primo processo fu condannata a 26 anni di reclusione (Aitken \& Taroni, 2004, pp. 128, 211-213). Il principale argomento che ha portato alla condanna è stato la valutazione - da parte di un professore di pediatria - della probabilità di morte improvvisa, per cause naturali, nei primi mesi di vita; tale probabilità è stata stimata, sulla base di recenti statistiche, in 1/8.500. La probabilità di due morti per SIDS nella stessa famiglia è quindi stata stimata col quadrato di tale valore (assumendo, in modo inappropriato, l'indipendenza stocastica dei due eventi; ma questo fatto non verrà qui discusso). La probabilità delle due morti è quindi stata stimata come $(1 / 8.500)^{2}=0,000000013$, una probabilità microscopica che ha portato 
il giudice nel primo processo a valutare come praticamente impossibile la morte naturale dei due figli. $\mathrm{Ma}$, come sappiamo, da una probabilità molto piccola - in sé e per sé - non si può derivare la produzione non casuale dell'evento associato.

Il giudice del processo di appello (gennaio 2003), che ha portato alla assoluzione di Sally Clark, ha basato sostanzialmente la sua decisione su elaborazioni statistiche di tipo bayesiano. La probabilità di omicidio di un bambino nel primo anno di vita è stata stimata (su dati UK) in circa 1/92.000; assumendo l'indipendenza fra gli eventi, per due omicidi la probabilità a priori di colpevolezza è $P(G)=(1 / 92.000)^{2}$, mentre la probabilità di innocenza è $P(\bar{G})=(1 / 8.500)^{2}$. Riprendiamo la formula di Bayes, nella forma odds ratio del par. 3 :

$$
\frac{P(G \mid E)}{P(\bar{G} \mid E)}=\frac{P(G)}{P(\bar{G})} \times \frac{P(E \mid G)}{P(E \mid \bar{G})} .
$$

Tenuto conto che $P(E \mid G)=P(E \mid \bar{G})=1$ (sia l'ipotesi di colpevolezza sia l'ipotesi di innocenza spiegano completamente le due morti), il suddetto rapporto si riduce a $P(G) / P(\bar{G}) \approx 0,0085$; ne deriva che $P(\bar{G} \mid E) \approx 0,99$, ovvero quasi certamente Sally Clark è innocente!

\section{I TEST DI IPOTESI}

L'impostazione bayesiana trova difficoltà applicative quando le probabilità in gioco sono eccessivamente imprecise; questo accade soprattutto per le c.d. probabilità a priori (probabilità delle cause). Vi è inoltre un tipo di applicazione (nei processi civili e penali) in cui l'approccio bayesiano è del tutto assente (anche se teoricamente ammissibile). Questo tipo di inferenza statistica ha riguardato in particolare, in moltissimi processi: (a) la discriminazione nei rapporti di lavoro, (b) la mortalità differenziale di un gruppo di lavoratori (rispetto alla popolazione generale), dovuta alle particolari condizioni del processo produttivo. Tali applicazioni, e molte altre similari, sono caratterizzate (1) da una valutazione statistica da associare a un campione più o meno ampio di individui, (2) dall'esistenza di norme giuridiche da rispettare, e/o di una popolazione di riferimento con la quale confrontare il campione disponibile.

Nei casi ora accennati si ricorre usualmente a una diversa impostazione: l'impostazione dei test di ipotesi di Fisher-Neyman-Pearson. 
In tale impostazione:

- non si assumono probabilità a priori,

- si lavora solo sulle verosimiglianze, e solo se sono sostanzialmente oggettive.

Ad esempio, riguardo ai rischi di esposizione a un agente tossico dei lavoratori di una azienda $Z$ (es. settore chimico), si confronta l'incidenza di una data malattia (o causa di morte) $M$ fra i lavoratori di $Z$ e la popolazione in generale, o con i lavoratori di un'azienda meccanica (ad esempio). Si accetta l'ipotesi $H_{0}$ (c.d. ipotesi nulla) di nessun effetto se la proporzione di $M$ fra i lavoratori di $Z$ è prossima (in senso probabilistico) a quella del gruppo di controllo.

In questa impostazione è essenziale controllare le due probabilità di errore:

$\alpha=$ probabilità di colpevolezza in caso di innocenza, $\beta=$ probabilità di innocenza in caso di colpevolezza.

Nel processo civile sembra equo mettere i due tipi di errore sullo stesso piano (cioè le due probabilità di errore dovrebbero essere molto prossime). Al contrario, nel processo penale prevale l'interesse a non condannare un innocente: è perciò ragionevole utilizzare in ogni caso una probabilità $\alpha$ molto piccola, e tipicamente molto minore di $\beta$. Si osservi che tali probabilità di errore non possono essere entrambe ridotte (possibilmente a valori molto piccoli) a parità delle informazioni campionarie: se si riduce una delle due probabilità di errore, l'altra deve aumentare; il test deve quindi essere opportunamente calibrato sulle probabilità di errore, e quindi sulle conseguenze - solitamente differenziate - di tali errori.

\section{RIFERIMENTI BIBLIOGRAFICI}

Aitken \& Taroni 2004 C.G.G Aitken \& F. Taroni, Statistics and the evaluation of evidence for forensic scientists, Chichester, Wiley, 2004.

Copi 1964 I. Copi, Introduzione alla logica, Bologna, Il Mulino.

Frosini 2002 B.V. Frosini, Le prove statistiche nel processo civile e nel processo penale, Milano, Giuffré.

Frosini 2009 B.V. Frosini, Metodi statistici, Roma, Carocci.

Stella 2001 F. Stella, Giustizia e modernità, Milano, Giuffré. 